Cahiers
de la Recherche
surles Droits

Cahiers de la recherche sur les droits

fondamentaux

$5 \mid 2006$

L'enfant

\title{
Le système africain de protection des droits de l'enfant
}

Exigences universelles et prétentions africaines

Jean-Didier Boukongou

\section{CpenEdition}

Journals

Édition électronique

URL : https://journals.openedition.org/crdf/7187

DOI : $10.4000 /$ crdf. 7187

ISSN : 2264-1246

Éditeur

Presses universitaires de Caen

Édition imprimée

Date de publication : 31 décembre 2006

Pagination : $97-108$

ISBN : 978-2-84133-277-9

ISSN : $1634-8842$

Référence électronique

Jean-Didier Boukongou, "Le système africain de protection des droits de l'enfant », Cahiers de la

recherche sur les droits fondamentaux [En ligne], 5 | 2006, mis en ligne le 15 décembre 2020, consulté le

14 novembre 2022. URL : http://journals.openedition.org/crdf/7187 ; DOI : https://doi.org/10.4000/ crdf.7187 


\title{
Le système africain de protection des droits de l'enfant Exigences universelles et prétentions africaines
}

\author{
Jean-Didier BOUKONGOU \\ Professeur \\ Université catholique d'Afrique centrale
}

I. Une convention progressiste

A. La difficile mise en lumière des droits de l'enfant en Afrique

1. Les articulations complexes des droits africains de l'enfant

2. Une protection régionale revendiquée

B. Prétention progressiste et adéquation socioculturelle

1. Une adhésion à la civilisation internationale des droits de l'homme

2. L'hypothèque du particularisme juridique africain

II. Un progrès incertain

A. Un chantier institutionnel en quête de légitimité et d'attractivité

1. Le Comité africain d'experts sur les droits et le bien-être de l'enfant

a. Le statut du Comité d'experts

b. La saisine du Comité d'experts

2. Les articulations avec les autres mécanismes de protection des droits de l'enfant

a. L'articulation avec la Commission et la Cour africaines des droits de l'homme

b. L'articulation avec le Comité des droits de l'enfant des Nations Unies

B. Un dispositif aux capacités et perspectives limitées

1. L'action entravée du Comité des experts

2. Le caractère titanesque des défis africains en matière de protection de l'enfant

Partout en Afrique, des enfants sont victimes de violences et abus multiples qui s'inscrivent dans des contextes économiques, socioculturels et politiques particuliers. Les agressions physiques, sexuelles et psychologiques subies par les enfants dans des situations de paix ou de guerre, dans leur environnement familial ou communautaire, constituent des obstacles à leur survie et à leur développement harmonieux. Au vu des images diffusées par les châ̂- nes internationales de télévision à propos des enfants mal nourris et malades au Soudan, à l'est du Congo (RDC), en Somalie, au Niger et ailleurs en Afrique, il peut paraître superflu de proposer une lecture critique d'une Charte africaine des droits et du bien-être de l'enfant (CADBE) ${ }^{1}$. Dès lors, toute réflexion juridique sur une telle problématique implique un effort de contrition morale et une distanciation stoïque pour gloser sur une construction

1. Adoptée par la $26^{\mathrm{e}}$ conférence des chefs d'État et de gouvernement de l'Organisation de l'unité africaine (OUA, devenue Union africaine, UA) à AddisAbeba (Éthiopie) le 11 juillet 1990 et entrée en vigueur le 29 novembre 1999, le texte est disponible sur le site http://www.africa-union.org. 
juridique en quête de légitimité, d'effectivité et d'attractivité.

Il aurait été plus commode de se limiter à une approche comparative des droits fondamentaux de l'enfant dans les divers systèmes nationaux africains, en insistant sur les droits de l'enfant dans la vie familiale (parents, frères et sœurs et ascendants) ou à l'égard de l'État (santé, éducation, etc. $)^{2}$. Une telle approche n'a cependant une crédibilité que dans des sociétés où la guerre, la maladie et la misère sont devenues de lointains souvenirs, sinon des accidents de parcours. Dans le contexte africain, l'enfant est au cœur d'une pauvreté et d'une insécurité routinières et persistantes. Malgré les affichages symboliques, l'exaltation de l'enfant comme « richesse commune » masque le sort déplorable qui lui est réservé dans des sociétés qui bafouent quotidiennement sa dignité humaine : écoles vétustes ou inexistantes, hôpitaux mouroirs, logements insalubres, migrations forcées, violences de toutes sortes, viols et abus sexuels, mutilations génitales, enrôlement dans les conflits, travail pénible ou forcé, esclavage, malnutrition, etc. Cette liste non exhaustive pourrait être utilement complétée par celle des pratiques culturelles négatives dont certains africanistes persistent à les classer dans le sarcophage des coutumes africaines.

Il aurait été tout aussi gratifiant d'épiloguer sur l'irradiation des systèmes africains par les normes internationales, la conspiration générale des droits internes par le droit international, et en particulier la Convention des Nations Unies sur les droits de l'enfant (CIDE). L'évocation des réformes législatives initiées ici et là, et les débats sur les rapports établis par les États selon les exigences de la convention de New York, auraient permis d'énoncer que la protection des droits de l'enfant progresse en Afrique, nonobstant les images médiatiques ponctuelles qui occultent la situation de la grande masse des enfants africains qui vont régulièrement à l'école et sont soignés selon les possibilités qu'offrent les systèmes sanitaires locaux. Il eût été possible d'en tirer quelques conclusions réconfortantes sur les progrès advenus en Afrique depuis 1989 et les obstacles à surmonter inéluctablement avec l'appui de la communauté internationale. Une telle approche trouverait de nombreux rapports d'experts sur lesquels nous pourrions nous appuyer pour étayer une argumentation juridique légitimant le progrès du droit international malgré quelques lacunes partielles. La marche triomphale de l'universalité des droits de l'homme s'en trouverait préservée et renforcée.

Sans sous-estimer l'intérêt de telles approches, nous avons plutôt choisi de traiter d'une perspective juridique latente, celle d'un objet juridique dont la réalité institutionnelle est précaire, même si son avènement a été salué avec enthousiasme comme la réalisation d'une ambition humaniste et d'une démarche progressiste dans le contexte africain. Progrès qui reste toutefois incertain face au caractère titanesque des défis à relever par rapport à la situation des droits de l'enfant en Afrique. Seize ans après son adoption, la CADBE apparaît comme une manifestation de la place croissante qu'occupe la protection des droits de l'enfant dans les préoccupations des États africains $^{3}$. Elle constitue une illustration de l'intégration des États africains dans l'évolution du droit international des droits de l'homme. Cependant, le véritable impact du système africain de protection des droits de l'enfant est encore indéterminé et dépendra notamment de la manière dont le chantier institutionnel ouvert sera réalisé et de son articulation avec les procédures préexistantes, tant au sein de l'UA que des Nations Unies.

\section{Une convention progressiste}

Devant la difficulté d'intégrer certaines préoccupations africaines dans la corbeille de la CIDE, les États africains ont choisi de renforcer les droits de l'enfant, en «prenant en considération les vertus de leur héritage culturel, leur passé historique et les valeurs de la civilisation africaine $»^{4}$. Malgré les difficultés traditionnelles à trouver une cohérence certaine autour d'un catalogue complet des droits de l'enfant, le but de la CADBE est d'apporter une protection régionale supplémentaire à celle apportée par la CIDE.

\section{A. La difficile mise en lumière des droits de l'enfant en Afrique}

Les droits de l'enfant proclamés dans les législations africaines montrent les articulations complexes entre les coutumes locales et le droit moderne écrit, ce qui pourrait expliquer le besoin d'une protection régionale plus avantageuse.

\section{Les articulations complexes des droits africains de l'enfant}

Les droits nationaux africains étant inspirés des systèmes juridiques des anciennes puissances coloniales, il y a souvent peu d'intérêt à mener une approche comparatiste, sinon pour tirer la conclusion de leur décalage par rapport aux réalités locales et de leur cristallisation par rapport aux évolutions rapides de la protection de l'enfance sur le plan international. Bien souvent l'objet des incantations anhistoriques, l'articulation entre les coutumes

\footnotetext{
2. J.-D. Boukongou, «Vie familiale comme lieu d'exercice des droits fondamentaux : lecture des pratiques africaines », in Le Défi des droits fondamentaux G. Otis et J.-Y. Morin (dir.), Bruxelles, Bruylant, 2000; A. Lamboley, «Les droits fondamentaux de l'enfant», in Libertés et droits fondamentaux, R. Cabrillac, M.-A. Frison-Roche, T. Revet (dir.), $12^{\mathrm{e}}$ éd., Paris, Dalloz, 2006, p. 253-282.

3. A.-D. Olinga, «La Charte africaine sur les droits et le bien-être de l'enfant: essai de présentation », Penant, n 820, 106 année, 1996, p. 53-68; F. Bugnion, "Les enfants soldats, le droit international humanitaire et la Charte africaine des droits et du bien-être de l'enfant », African Journal of International and Comparative Law, vol. 12, 2000, p. 262-275; M. Affa’a Mindzié, «La Charte africaine sur les droits et le bien-être de l'enfant », sur le site: http://www.droitshumains.org/Biblio/Txt_Afr/instr_mindzie.htm (consulté le 30 août 2006).

4. Préambule de la CADBE.
} 
africaines et le droit moderne écrit d'imposition coloniale rend difficile la compréhension de la problématique de la protection de l'enfant en Afrique. Les illustrations sont nombreuses. Par exemple, l'article 180 du Code pénal du Cameroun sanctionne le défaut de paiement de la pension alimentaire alors que de nombreuses mères célibataires éprouvent des difficultés pour recouvrer une pension alimentaire pour les enfants naturels non reconnus par leurs géniteurs. Il convient de relever que certains pères sont rétifs au versement de la pension alimentaire parce qu'elle est prétendument utilisée pour élever les enfants autres que les leurs, alors qu'eux-mêmes délaissent leur famille au profit des concubines. Le Code pénal Camerounais, en ses articles 179, 180, 355 et 358, punit respectivement la non-représentation de l'enfant à celui à qui la garde a été confiée par décision judiciaire, le refus de paiement de la pension alimentaire et l'abandon du foyer familial. Ces dispositions ne sont pas souvent appliquées avec rigueur.

L'État camerounais a intégré dans sa législation la disposition de l'article 7 de la CIDE qui stipule que «l'enfant est enregistré aussitôt sa naissance et a, dès celle-ci, le droit à un nom, le droit d'acquérir une nationalité». Néanmoins, il faut relever au niveau de la constatation juridique des naissances l'absence de données statistiques sur les enregistrements des naissances. Les actes d'état civil ne sont pas tous fiables. Au niveau de l'attribution du nom, la pratique qui consiste à attribuer des noms fantaisistes aux enfants devient courante.

À l'instar de nombreux pays africains, la législation congolaise (République démocratique du Congo) sur la définition de l'enfant maintient une insécurité juridique du fait de la contradiction entre le droit écrit et les coutumes locales ${ }^{5}$. L'enfant congolais se définit selon les aspects civil et pénal. En matière civile, l'enfant est la personne humaine de moins de 18 ans tandis qu'en matière pénale, c'est celui qui a moins de 16 ans. Toutefois, l'inexistence de l'état civil rend difficile la détermination de l'âge de la personne à considérer. En effet, beaucoup d'enfants ne connaissent pas leur âge. Cette ignorance est même « tolérée» par le législateur qui, dans certaines dispositions légales, utilise les expressions telles que «âgés de [...] ou apparemment âgé de» qui s'analysent en fin de compte comme un fourre-tout, ce qui permet à certains services de l'État de considérer comme enfant ou comme adulte celui qu'on veut comme tel.

Par ailleurs, le même Code de la famille interdit à toute personne qui, en vertu de la loi ou de la coutume, a le droit de garde sur une fille n'ayant pas atteint l'âge de la puberté, de la remettre en mariage ou en vue d'un mariage. Or, la puberté est fixée à 14 ans, autrement dit à 14 ans la fillette peut être prise en mariage. Le même Code reconnaît en son article 317 la coresponsabilité du père et de la mère dans l'exercice de l'autorité parentale. Cependant, il existe des coutumes locales qui admettent la distinction nette entre le père biologique de l'enfant et celui qui doit exercer sur lui l'autorité parentale, à savoir l'oncle mater- nel (frère de la mère de l'enfant), alors que le père biologique est en vie. C'est le cas des coutumes issues des cultures matrilinéaires, comme chez les Bakongo du sud-ouest du pays. La conséquence est que l'échec de l'éducation des enfants est de la responsabilité des oncles et non de leur père qui souvent ne les considère pas comme des membres de sa famille. Il y a donc, dans certains cas, une fiction de l'autorité parentale entre l'oncle qui a l'autorité coutumière et effective et le père qui a l'autorité légale et fictive. Toutefois, l'un ou l'autre peuvent faire usage des dispositions de l'article 326 du même Code qui permet à celui qui a l'autorité parentale d'infliger à l'enfant réprimande et correction dans une mesure compatible avec son âge et l'amendement de sa conduite. Étant donné que la notion de correction s'apprécie selon celui qui l'inflige, elle aboutit souvent aux violences physiques, à la privation prolongée de nourriture, des habits ou des jouets.

Quant au principe de participation, les problèmes démocratiques que rencontrent les pays africains se répercutent au niveau des droits de l'enfant. Le Parlement des enfants de la Côte d'Ivoire mis en place depuis 1992 a besoin de renouveler les membres de son Bureau exécutif qui, depuis longtemps, ont terminé leur mandat et dont les membres ont atteint l'âge de la majorité. Il arrive que le parti au pouvoir instrumentalise cette représentation des enfants par un bourrage idéologique inconséquent.

La question de l'adoption internationale des enfants soulève aussi de nombreuses interrogations en Afrique. Les États africains n'offrent que peu de possibilités aux procédures d'adoption internationale. Le Code de la famille au Congo (Brazzaville) exige que le couple demandeur soit marié depuis au moins cinq ans et qu'il n'ait pas d'enfant biologique, sauf dispense du ministère de la Justice. Si l'Éthiopie confie depuis longtemps des enfants à des familles étrangères (depuis l'époque de la grande famine), les autres pays ne se sont ouverts à l'adoption internationale que récemment. En effet, la prise en charge traditionnelle des enfants au sein de la famille africaine élargie (oncles, tantes...) et du village a longtemps permis de trouver des solutions familiales localement. Mais le sida, les conflits et la pauvreté ont conduit certains pays à chercher, hors de leurs frontières, des familles pour leurs enfants qui en sont privés. Dans le cas des enfants africains adoptés en France, les principaux pays d'origine en 2003 étaient: Madagascar (325 enfants), l'Éthiopie (217), le Mali (132), le Burkina Faso (6o) et Djibouti, le Congo, le Togo (moins de 60 chacun).

Au Tchad, des enfants sont aujourd'hui encore vendus et réduits en esclavage. Des adolescents vendus par leurs parents pour 10 à 15000 francs Cfa (entre 15 et 23 euros), à des éleveurs arabes venus du nord. D'autres sont échangés contre un veau. La pratique des enfants bouviers a commencé dans les années 1970, en période de grande sécheresse au Sahel. À cause de la pauvreté, elle s'est développée dans toute la partie sud du pays. Certaines familles préfèrent souvent se débarrasser de leurs enfants valides 
pour avoir moins de bouches à nourrir. Selon des agences humanitaires basées au Tchad, on compte aujourd'hui encore quelque 2000 enfants âgés à peine de 8 ans, vendus par leurs parents. Ils sont réduits à l'état d'esclavage par leurs maîtres. Pour certaines ONG, ce chiffre ne reflète toutefois pas la réalité, d'autant moins que nombre d'enfants meurent pour cause de mauvais traitements. L'entreprise de récupération des enfants s'avère parfois risquée : des éleveurs arabes attaquent les personnes qui tentent de leur retirer des enfants, estimant être dans leur droit, du fait de les « avoir acheté » et donc de pouvoir en « disposer».

Au Bénin, le Code du travail (Ordonnance n ${ }^{\circ} 33$ / / PR / MFPTTdu 28 septembre 1967) limite l'âge minimum d'admission à un emploi à 14 ans, mais il existe des arrêtés de la période coloniale qui sont encore en vigueur et qui définissent des dérogations par rapport à l'âge d'admission à l'emploi qui peut alors baisser jusqu'à 12 ans. La plus grande frange des enfants travailleurs est essentiellement constituée de mineurs déscolarisés ou non scolarisés. La forme de travail des enfants qui est le plus d'actualité est celle relative aux enfants placés appelés «Vidomègon ». Le Vidomègon est un enfant placé auprès d'un tiers par ses parents ou par une personne appelée placeur ou intermédiaire dans le but de lui faire acquérir une éducation ou de le faire travailler. Autrefois considérée comme une marque de solidarité traditionnelle entre parents et membres d'une même famille, Le Vidomègon a été travesti aujourd'hui en placement d'enfant lucratif par l'intermédiaire des tiers qui bénéficient d'une rémunération. Le phénomène a pris désormais les dimensions d'une véritable entreprise gérée par des personnes organisées en réseaux criminels dont les activités de trafic des êtres humains sont devenues internationales.

L'histoire du bateau nigérian Etireno, en avril 2001, a montré la complexité de ce trafic. Dans la pratique du trafic d'enfants, il y a des pays pourvoyeurs, des pays récepteurs et des pays qui sont à la fois pourvoyeurs et récepteurs. Le recours abusif à une main-d'œuvre domestique corvéable et bon marché compte tenu de l'évolution des conditions socio-économiques de la population tant urbaine que rurale, la multiplicité des intermédiaires attirés par le gain facile et la quasi-absence de risque doublée d'une certaine complicité sociale, expliquent la recrudescence de la pratique. Les trafics internes sont animés comme une activité du secteur informel; les trafics transfrontaliers procèdent d'une organisation en filière relevant davantage du crime organisé. À l'absence de mesures pré- ventives et coercitives s'ajoute la perméabilité des frontières en raison des insuffisances normatives et institutionnelles. Plusieurs conventions internationales prohibent le trafic des enfants. Elles ont été ratifiées par la majorité des pays africains. Les principales conventions qui font référence au travail forcé et au trafic sont : la Convention $\mathrm{n}^{\circ} 29$ de l'Organisation internationale de travail (OIT) sur le travail forcé (de 1930), la Convention supplémentaire sur l'abolition de l'esclavage, les institutions et les pratiques similaires à l'esclavage (de 1956), la Convention relative aux droits de l'enfant (de 1989), notamment son article 35, la Charte africaine des droits et du bien-être de l'enfant (articles 24 et 29), et la Convention $n^{\circ} 182$ de l'OIT relative aux pires formes de travail des enfants (de 1999).

La manifestation immédiate de la pratique est l'exploitation illégale et abusive à la fois de l'enfant en tant qu'être humain, de sa force de travail et de son travail luimême. L'exploitation dont il est question n'est pas seulement une exploitation économique de l'enfant. Elle est aussi une escroquerie morale qui prend l'allure d'un vol de l'enfance de la victime, avec le plus souvent le consentement et même la complicité de ses parents. Si le travail en milieu domestique ou le travail précoce en milieu formel est constaté comme la finalité la plus répandue du trafic, il existerait selon certains témoignages des pratiques socioreligieuses traditionnelles qui exigeraient $\mathrm{du}$ sang humain de jeune enfant ou ce qu'on désigne communément par l'expression macabre de "pièces détachées ».

Ces articulations complexes sont porteuses de nombreuses discontinuités qui résistent à la dynamique de conspiration juridique progressivement induite par la CIDE et ses deux protocoles facultatifs.

\section{Une protection régionale revendiquée}

La compréhension du système régional spécifique consacrant l'enfant africain comme une préoccupation spéciale doit se faire à la lumière du système africain général de protection des droits de l'homme ${ }^{6}$. La prolifération internationale des mécanismes de protection des droits de l'homme participe de la construction d'un nouvel «ordre mondial des droits de l'homme ${ }^{7}$. Le système régional africain des droits de l'homme est souvent décrit par sa complexité normative et son inefficacité procédurale ${ }^{8}$. Perçu plutôt à l'origine comme « un nouvel ordre humanitaire régional en Afrique ${ }^{9}$ que comme un système juridictionnel, la Charte africaine des droits de l'homme et des peuples est l'instrument principal et général de protection des droits de l'homme en Afrique ${ }^{10}$, même si ledit système

6. J.-D. Boukongou, «L'application de la Charte africaine des droits de l'homme et des peuples par les autorités nationales en Afrique centrale», in L'Application nationale de la Charte africaine des droits de l'homme et des peuples, J.-F. Flauss, E. Lambert-Abdelgawad (dir.), Bruxelles, Bruylant Nemesis (Droit et justice; 54), 2004, p. 123-160.

7. J. Mourgeon, «Développement et droits de l'homme», in Aspects du système des Nations Unies dans le cadre de l'idée d'un nouvel ordre mondial, Y. Daudet (dir.), Paris, Pedone, 1992, p. 188; G. Cohen-Jonathan, «L'évolution du droit international des droits de l'homme », in Mélanges offerts à Hubert Thierry. L'évolution du droit international, Paris, Pedone, 1998, p. 107-125.

8. M. Mubiala, Le Système régional africain de protection des droits de l'homme, Bruxelles, Bruylant, 2005; A.-D. Olinga, «L'effectivité de la Charte africaine des droits de l'homme et des peuples ", Revue Afrique 2000, $\mathrm{n}^{\circ} 27$ / 28, avril-octobre 1997, p. 171; E.-A. Ankumah, La Commission africaine des droits de l'homme et des peuples. Pratiques et procédures, Londres, SADIC, 1995.

9. F. Ouguergouz, La Charte africaine des droits de l'homme et des peuples. Une approche juridique des droits de l'homme entre tradition et modernité, Paris, PUF, 1993, p. 393.

10. Recueil juridique des droits de l'homme en Afrique (1996-20oo), P. Tavernier (éd.), Bruxelles, Bruylant (Collection du Credho ; vol. 2), 2002. 
est fondé, selon les articles 60 et 61 de la dite Charte, sur l'application du droit international relatif aux droits de l'homme et sur les autres textes régionaux africains, dont la CADBE. Selon ses «pères fondateurs », le système africain devrait être non seulement en adéquation avec son environnement politique et socioculturel, mais il devrait aussi avoir une cohérence juridique qui découle d'une « conception globalisante des droits de l'homme ${ }^{11}$ prescrivant d'une part l'équilibre entre les droits et les devoirs, entre l'individu et la communauté, et d'autre part privilégiant la conciliation au contentieux des droits de l'homme au motif que dans la conception africaine « les conflits sont tranchés non pas par une procédure contentieuse mais par la conciliation [...] qui aboutit à un consensus et qui ne fait ni vainqueur ni vaincu ${ }^{12}$.

Malgré le fait que tous les États africains, à l'exception de la Somalie, soient parties à la Convention des Nations Unies relative aux droits de l'enfant, seuls 38 États, sur les 53 membres de l'Union africaine (UA), ont ratifié la CADBE au 31 décembre 2005, ce qui dénote du peu d'empressement de la part des États africains à souscrire aux particularités socioculturelles tant revendiquées sur le théâtre diplomatique international ${ }^{13}$. Et pourtant l'existence de cette CADBE fut justifiée par la non-prise en compte dans la CIDE de certaines questions particulières touchant des aspects spécifiques aux droits de l'enfant africain ; méprise qui serait due à l'objectif suprême qui était d'arriver à un compromis universel dans l'instrument onusien.

Une étude des dispositions de la CADBE fait apparaître, à côté des droits également protégés par la CIDE, certains droits dont la protection constitue un progrès dans le contexte africain et d'autres droits qui, malheureusement, laissent apparaître des formulations lacunaires et ambiguës au point de conforter le scepticisme quant à l'efficacité d'un tel instrument. De même, le Comité africain d'experts sur les droits et le bien-être de l'enfant prévu par la CADBE est un progrès institutionnel dont les capacités opérationnelles et juridictionnelles font l'objet d'une maturation latente au sein du système global de l'Union africaine. Le fonctionnement du Comité et ses résultats seront-ils efficaces pour attirer un grand nombre de justiciables? Ce système n'aurait-il que le mérite d'exister ou peut-il contribuer à une amélioration substantielle des droits de l'enfant en Afrique?

Il faut cependant faire remarquer que les États africains se sont fortement engagés à produire ou à souscrire aux engagements internationaux en matière de droits de l'homme. Non pas que leur bonne foi en la matière soit contestable, mais s'y refuser équivaut presque à se mettre au ban de la communauté internationale. Formuler des réserves explicites sur tel ou tel aspect de protection des droits de l'enfant reviendrait à se montrer soi-même du doigt. Il est donc préférable de signer les textes les plus sublimes, de prendre les engagements les plus contraignants en apparence avec la ferme intention de n'en tenir aucun compte. Telle est la leçon à tirer de la pratique de beaucoup d'États africains depuis leur indépendance. Bien souvent, le combat pour les droits de l'homme au niveau international n'est qu'une entreprise d'attractivité diplomatique permettant d'être dispensé, dans l'immédiat, de rendre compte des violations routinières de la dignité humaine.

Dans le même sens les thuriféraires d'un « droit international africain » encensent les gouvernements qui ratifient à tour de bras les conventions internationales au motif qu'ils témoigneraient ainsi de leur adhésion aux exigences universelles de la communauté internationale. Les analyses juridiques qui en découlent exaltent les révolutions juridiques qui modifieraient substantiellement les pratiques locales. Cependant, de telles incantations et le verbalisme panafricain sur le bien-être de l'enfant n'ont pas réussi, à ce jour, à combler le fossé grandissant entre une jeunesse africaine désemparée par l'aggravation du mal-être et les préconisations millénaristes de la communauté internationale.

\section{B. Prétention progressiste et adéquation socioculturelle}

Sans une prise en charge spécifique de la situation de l'enfant en Afrique par la CIDE, face au refus de la communauté internationale de créer un déséquilibre au profit des particularismes régionaux, la communauté africaine se trouvait devant un problème juridique et moral majeur, qui ne pouvait demeurer en l'état, sans porter préjudice à l'amélioration de la condition juridique de l'enfant en Afrique.

\section{Une adhésion à la civilisation internationale des droits de l'homme}

Malgré les postures culturalistes et les rodomontades diplomatiques, il aurait été miraculeux que les États africains produisent un instrument régional qui se démarque complètement de la CIDE à laquelle leur adhésion était certaine, sans qu'il soit besoin d'exciper des pressions internationales toujours mises de manière quasi religieuse au fronton des ratifications africaines par les thuriféraires des droits de l'homme. Au-delà de ses imperfections progressivement amendées par les protocoles facultatifs, la Convention de New York est un compris consensuel auquel ne pouvaient se soustraire ceux des États africains qui s'estimaient avoir été oubliés dans le processus d'enfantement de la CIDE. Les deux conventions (CIDE et CADBE) ont de nombreuses dispositions en commun.

11. Voir Y. Eteka, La Charte africaine des droits de l'homme et des peuples, Paris, L'Harmattan, 1996, p. 370.

12. K. Mbaye, Les Droits de l'homme en Afrique, $2^{\mathrm{e}}$ éd., Paris, Pedone, 2002, p. 28 ; C.-M. Eya Nchama, Développement et droits de l'homme en Afrique, Paris, Publisud, 1991; T. Matala Kabangu, «Les droits de l'homme en Afrique : énoncé, garanties et obligations », in Les Droits de l'homme à l’aube du XXI siècle. Karel Vasak Amicorum Liber, Bruxelles, Bruylant, 1999, p. 633-654.

13. L. Sindjoun, «La civilisation internationale des mœurs : éléments pour une sociologie de l'idéalisme structurel dans les relations internationales», Études internationales, vol. $27, \mathrm{n}^{\circ}$ 4, décembre 1996, p. 848. 
La CADBE s'inspire de la CIDE et les deux instruments partagent des principes fondamentaux de l'application des droits de l'enfant reconnus au plan universel, tels que le principe de non-discrimination, de respect de l'intérêt supérieur de l'enfant, de participation des enfants, de survie et de développement de l'enfant et de prise en compte de l'évolution des capacités de l'enfant. Ces principes enveloppent l'ensemble des droits civils et politiques, des droits économiques, sociaux et culturels, ainsi que certains droits de protection spécifiques aux enfants. Il s'agit, par exemple, du droit à la vie et l'interdiction de prononcer la peine de mort contre des enfants, du droit à un nom, à l'enregistrement dès la naissance, du droit à une nationalité, de la liberté d'expression, d'association, de pensée, de conscience et de religion ou encore de la protection de la vie privée. Sont également protégés le droit à l'éducation, aux loisirs, aux activités culturelles et récréatives, la protection des enfants handicapés ou le droit de l'enfant à la santé et aux services médicaux. Et enfin, la CADBE prévoit des droits spécifiques de protection des enfants réfugiés, des enfants soumis à des procédures d'adoption et des enfants séparés de leurs parents. De même, les enfants doivent être protégés contre l'exploitation économique, contre toutes formes d'abus et de mauvais traitements, contre l'exploitation sexuelle, l'usage et le trafic de stupéfiants ou encore la vente, la traite et l'enlèvement d'enfants.

Cependant la CADBE ne serait qu'une œuvre de Sisyphe si elle devait se contenter d'une reprise à l'identique de la CIDE. D'où, selon certains auteurs, les progrès réalisés dans divers domaines. C'est par exemple le cas de la définition de l'enfant. Durant les travaux de rédaction de la CIDE, la définition de l'enfant a divisé les représentants des diverses conceptions religieuses et philosophiques, tant par rapport au début de l'enfance qu'à sa fin. La pluralité des âges de majorité en fonction des circonstances ou des activités donne l'impression d'une majorité à géométrie variable.

Dans le cadre africain, la CADBE tranche le débat en précisant à l'article 2 que l'enfant est «tout être humain âgé de moins de 18 ans ». Cette disposition permet d'octroyer à toute personne de moins de 18 ans la protection spéciale qui lui est due, en dépit d'une législation qui fixerait plus tôt, l'âge de la majorité. Cette affirmation de principe rentre cependant en conflit avec certains droits nationaux qui persistent dans les différentiations contextuelles quant à la détermination du sujet enfant. Ce progrès s'oppose particulièrement à la velléité de certains gouvernements à enrôler les enfants dans les forces armées et les organisations paramilitaires prolifiques en Afrique. La CIDE, par la suite complétée par un Protocole facultatif adopté en la matière, n'avait pas interdit la participation aux conflits des enfants de plus de 15 ans. La CADBE précise que les États parties prendront «toutes les mesures nécessaires pour veiller à ce qu'aucun enfant ne prenne directement part aux hostilités et en particulier, à ce qu'aucun enfant ne soit enrôlé sous les drapeaux ».

Outre cet exemple principal, la CADBE contient diverses autres stipulations qui démontrent le caractère progressiste de la protection des droits de l'enfant en Afrique.
C'est le cas de l'article 21 qui interdit les pratiques culturelles et sociales néfastes au bien-être, à la dignité, à la croissance et au développement normal de l'enfant, y compris les mariages d'enfants et les promesses d'enfants en mariage. Indépendamment de la CADBE, il existe un Comité interafricain (CIA) sur les pratiques traditionnelles depuis une vingtaine d'années. Selon le rapport présenté par ce comité à la cinquième réunion du Comité d'experts de la CADBE à Nairobi (Kenya) du 8-12 novembre 2004, les pratiques traditionnelles africaines sont basées sur les opinions, les croyances et les coutumes et de ce fait, elles pourraient soit être bénéfiques pour le groupe social, soit lui être nuisibles. Le CIA met l'accent sur la mutilation génitale féminine (MGF) en exposant les différentes sortes de mutilation, les complications qui découlent de cette pratique, les liens existant entre ces mutilations et la transmission du VIH. Il insiste sur l'importance de cette question au regard des droits de l'homme et considère que le problème de la MGF devrait être traité d'une manière globale dans le contexte de la tradition, de la culture, de la santé et des droits de l'homme.

La protection des enfants réfugiés et des enfants déplacés à l'intérieur d'un pays est aussi une préoccupation spécifique de la CADBE prévue à l'article 23. Il n'est pas superflu de rappeler que si les enfants sont enrôlés dans les conflits en Afrique, ils sont autant victimes des déplacements forcés provoqués par ces conflits. Une protection spécifique était donc nécessaire vu que le droit international est encore lacunaire sur cet aspect particulier contrairement à la protection internationale des réfugiés.

La CADBE a également tenu compte des réalités socioculturelles africaines avec des mesures spéciales, notamment en matière d'éducation, qui devront être prises en faveur des filles qui tomberaient enceintes avant la fin de leurs études. Il en est de même pour les enfants soumis à la discrimination (article 26), pour les enfants contraints à la mendicité (article 29) ou encore les enfants dont les mères purgeraient une peine d'emprisonnement (article 30). La CADBE constitue, au vu de l'ensemble de ces éléments juridiques, un progrès, malgré une hypothèque socioculturelle qui devrait être levée.

\section{L'hypothèque du particularisme juridique africain}

La quête du particularisme socioculturel a conduit les États africains à des prétentions juridiques ambiguës en ce qui concerne des devoirs que tout enfant aurait envers sa famille, la société, l'État et la communauté internationale. L'article 319 du Code de la famille du Congo (Brazzaville) dispose que «l'enfant, à tout âge, doit honneur et respect, aide et assistance à ses père et mère, aux collatéraux de ces derniers et à ses autres ascendants ». Il y a un réel malaise quant à la pertinence juridique de telles proclamations qui feignent de consacrer des normes dépourvues de toute considération pratique, même si leur utilité sociale peut être mise en exergue. Si les devoirs de l'enfant peuvent donner lieu à un comportement social exemplaire de la part de ce dernier, il reste qu'une persistante inquiétude juridique est manifeste quant à leur réelle juridicité 
et à leurs conséquences concrètes sur le plan procédural. Sans nier l'intérêt de l'argumentation suggérée par le président sénégalais Senghor et développée par le juge Kéba Mbaye $^{14}$ à propos de l'équilibre entre les droits et les devoirs dans la conception africaine des droits de l'homme, la vraie question que l'on pourrait se poser à propos des devoirs de l'enfant est celle de savoir comment s'assurer que ce dernier s'acquitterait de ses devoirs au titre de la CADBE ? Ou bien faut-il simplement en déduire que cette construction normative aléatoire ne témoigne que de la vertu pédagogique de la $\mathrm{CADBE}$, en quelque sorte des balises éthiques qui éclairent l'éducation des enfants en Afrique loin de toute pratique juridique concrète?

Le doyen Madiot s'est longuement interrogé sur la place des devoirs dans l'ordre juridique africain des droits de l'homme. Selon lui, les devoirs mettent «l'individu au service de la communauté et permettent de justifier toutes les oppressions ${ }^{15}$. La prescription des devoirs ne serait donc qu'une exaltation de l'arbitraire et une justification des doctrines développementalistes peu soucieuses des droits de l'individu. L'enfant serait sacrifié au profit soit d'un développement communautaire illusoire, soit astreint à un unanimisme sanguinaire qui a marqué de nombreux pouvoirs africains. Même le retour aux valeurs traditionnelles africaines ne serait qu'une façade qui conduirait à une impasse juridique ${ }^{16}$.

Certes en pratique, la Commission africaine des droits de l'homme et des peuples ne s'est pas heurtée à cette confusion entre les droits et les devoirs. Faut-il pour autant continuer à maintenir des prescriptions qui ne sont ni convaincantes, ni praticables au risque de proroger une suspicion légitime sur le camouflage de l'arbitraire? Un délestage normatif de la CADBE, comme de la Charte africaine des droits de l'homme et des peuples, semble nécessaire et opportun à propos des devoirs, d'autant plus que la doctrine est d'avis que cette consécration n'est plus véritablement valable dans le contexte actuel de renouveau démocratique et de la citoyenneté ${ }^{17}$. En définitive, à défaut d'une amélioration qualitative des instruments africains dans l'immédiat, il conviendrait provisoirement de s'abriter derrière la vénérable sagesse de René-Jean Dupuy : «On ne saurait opposer, dans l'absolu, communauté et individu [...]. La dialectique est celle de la communauté, sans laquelle l'homme est irresponsable, replié sur ses droits comme sur un patrimoine égoïstement gardé, et celle de l'homme, sans lequel la communauté devient une entité oppressive sinon meurtrière ${ }^{18}$. Cette question demeure d'actualité pour un texte proclamé afin de garantir aux enfants la protection spéciale qui leur est due, en raison de leur vulnérabilité.
Par ailleurs, on ne peut s'empêcher de constater les incertitudes juridiques qu'entretient la CADBE par rapport à la CIDE. Ces incertitudes concernent, par exemple, les développements relatifs aux enfants en conflit avec la loi et, plus particulièrement, les enfants privés de liberté. C'est ainsi que la CADBE ne contient pas de disposition stipulant expressément qu'aucun enfant ne saurait être privé de liberté de façon illégale ou arbitraire, ou que «l'arrestation, la détention ou l'emprisonnement d'un enfant doit être en conformité avec la loi, n'être qu'une mesure de dernier ressort, et être d'une durée aussi brève que possible », comme le précise l'article 37 b de la CIDE. De même sont absents des principes clés de l'administration de la justice pour les mineurs tels que : la légalité et la non-rétroactivité des peines et des délits, le principe selon lequel aucun enfant ne saurait être contraint de témoigner ou de s'avouer coupable, ou encore la nécessité de "prendre des mesures, chaque fois que cela est possible et souhaitable, pour traiter ces enfants sans recourir à la procédure judiciaire ». Ces incertitudes s'étendent aussi au droit de tout enfant à l'information ou à une protection sociale. Certes proclamer ces droits n'aurait pas conduit à leur satisfaction immédiate au regard des difficultés économiques et sociales persistantes en Afrique, mais il aurait mieux valu les énoncer au détriment des devoirs qui ne font qu'accentuer le caractère incertain du progrès revendiqué.

Enfin, ainsi que c'était déjà le cas pour la Charte africaine des droits de l'homme et des peuples, les clauses de limitation contenues dans la CADBE ne semblent pas présenter toutes les garanties d'un caractère nécessaire dans une société démocratique. En effet, l'exigence de légalité est la seule condition prévue et aucune mention n'est faite quant aux motifs de restriction habituellement retenus qui tiennent à la protection de l'ordre public, de la santé et de la morale publique, de la sécurité ou encore des droits et des libertés d'autrui. Comme la Commission africaine des droits de l'homme l'a déjà fait, il reviendra sans doute au Comité d'experts ou à la Cour africaine des droits de l'homme de préciser les conditions des restrictions que les États parties pourront apporter aux droits reconnus aux enfants en Afrique dans la CADBE.

\section{Un progrès incertain}

Superposition d'un quadruple catalogue formel de protection des droits de l'enfant en Afrique (coutumière, national, régional et international), toute divergence ne saurait être exclue entre ces divers ordres juridiques. Pour

14. F. Ouguergouz, La Charte africaine des droits de l'homme et des peuples..., p. 233-234.

15. Y. Madiot, Considérations sur les droits et devoirs de l'homme, Bruxelles, Bruylant, 1998, p. 126.

16. S. Kaba, L'Avenir des droits de l'homme en Afrique au XXI siècle, Dakar, Le nouvel horizon, 1996 ; J. Fierens, «La Charte africaine des droits de l'homme et des peuples ", RTDH, vol. 1, 1990, p. 235-248.

17. A.-D. Olinga, «L'impératif démocratique dans l'ordre régional africain », Revue de la Commission africaine des droits de l'homme et des peuples, vol. 8 , $\mathrm{n}^{\mathrm{o}} 1,1999, \mathrm{p} .55-76$.

18. R.-J. Dupuy, "Thème et variations sur le droit au développement", in Mélanges offerts à Charles Chaumont. Le droit des peuples à disposer d'euxmêmes, méthodes d'analyse du droit international, Paris, Pedone, 1984, p. 273. 
prévenir les tiraillements incessants, une position de principe semble se dégager des travaux du Comité d'experts de la CADBE sur une correspondance d'interprétation entre la CADBE et la CIDE, tout en préservant le fait que le droit régional africain accorde une protection plus étendue au regard des réalités africaines. Ce qui démontre que la CADBE devrait pouvoir s'intégrer harmonieusement dans les différents champs institutionnels préexistants, internes et internationaux, dans la mesure où elle va permettre de renforcer l'offre des droits et des garanties pour la protection de l'enfant en Afrique. Cependant, la complexité du système africain, les difficultés de son institutionnalisation, la multiplication des acteurs, les capacités limitées du système et les défis titanesques sur la situation de l'enfant en Afrique indiquent que ce progrès est incertain.

\section{A. Un chantier institutionnel en quête de légitimité et d'attractivité}

Il est légitime et opportun de s'interroger sur l'effectivité et l'attractivité de la CADBE. L'adéquation socioculturelle dont elle serait l'expression normative, et qui visait à compléter le système universel fondé sur la CIDE, pourrait-elle sanctuariser les droits de l'enfant en Afrique?

\section{Le Comité africain d'experts sur les droits et le bien-être de l'enfant}

Le Comité d'experts a un statut classique d'organe quasi juridictionnel de protection des droits de l'homme. Cependant, les conditions de sa saisine méritent d'être mieux précisées, faute de dispositions spécifiques sur les conditions de recevabilité des communications individuelles.

\section{a. Le statut du Comité d'experts}

L'article 32 la CADBE a créé le Comité d'experts, composé de onze membres siégeant à titre personnel et ayant les plus hautes qualités de moralité, d'intégrité, d'impartialité et de compétence pour toutes les questions concernant les droits et le bien-être de l'enfant. En vertu de l'article 11 (2) du Règlement intérieur adopté par le Comité, «l'état de membre du Comité africain est incompatible avec une activité susceptible de compromettre l'indépendance ou l'impartialité du membre, ou de porter préjudice à ses fonctions telles que travailler dans une organisation intergouvernementale, une institution de l'ONU ou occuper des fonctions de Ministre, de Vice-ministre, de député, d'ambassadeur ou tout autre poste comportant une affiliation politique». Ils sont élus pour 5 ans et, contrairement aux membres de la Commission africaine des droits de l'homme et des peuples, ils ne peuvent être réélus.

Le Comité d'experts est un organe de l'Union africaine. Conformément à l'article 34 de la CADBE, les premiers membres du Comité d'experts ont été élus par la $37^{\mathrm{e}}$ conférence des chefs d'État et de gouvernement de l'UA, tenue à Lusaka en Zambie en juillet 2001. Outre la question des incompatibilités avec le mandat d'expert, le Règlement intérieur du Comité précise l'organisation du
Bureau et du Secrétariat. Il faut toutefois remarquer que jusqu'à la date de $s a 7^{\mathrm{e}}$ session en décembre 2005, le Comité n'avait pas encore obtenu de la Conférence de l'Union africaine la nomination d'un secrétaire permanent, malgré l'ouverture du poste depuis de nombreux mois.

Au-delà de cette affirmation de son indépendance, on peut s'interroger sur le lien ombilical entre le Comité d'experts et la Commission des affaires sociales de l'UA. En effet, contrairement aux doctrines étatiques dominantes, la question des droits de l'enfant ne relève pas exclusivement des affaires sociales. Comme toute problématique des droits de l'homme, la vision sociale tend à dénaturer les objectifs de la CADBE. Il s'agit avant tout des droits, c'est-à-dire des revendications de type juridique qui ne se limitent pas à la sphère des compensations sociales. Cette tutelle organique est inadéquate dans la mesure où le Comité doit conforter son indépendance par rapport aux membres de la Commission de l'UA (organe politique par excellence) et aux représentants des États au sein de l'Union africaine. À défaut d'un secrétariat autonome, il serait plus judicieux de mutualiser les ressources du secrétariat de la Commission africaine des droits de l'homme et des peuples en attendant de pourvoir aux postes requis.

\section{b. La saisine du Comité d'experts}

Le mandat du Comité d'experts est précisé à l'article 42 de la CADBE. Il en ressort que le Comité a pour missions de promouvoir et protéger les droits consacrés dans la CADBE, d'en suivre l'application et veiller à leur respect, d'interpréter les dispositions de la CADBE à la demande des États parties, des institutions de l'UA ou de toute autre institution et, enfin, de s'acquitter de toute autre tâche qui pourrait lui être confiée par la conférence des chefs d'État et de gouvernement, par le président de la Commission de l'UA ou par tout autre organe de l'Organisation.

Les articles 43 à 45 de la CADBE énoncent les différentes compétences reconnues au Comité, afin de remplir les missions qui lui sont attribuées. En vertu de l'article 43, le Comité d'experts est compétent pour recevoir et examiner les rapports des États parties, sur les mesures qu'ils ont adoptées afin de rendre effectives les dispositions de la CADBE ainsi que sur les progrès réalisés dans l'exercice des droits proclamés. Les États ne s'empressent pas toujours de présenter leurs rapports malgré les nombreux rappels du Comité.

Le Comité d'experts a une compétence pour procéder aux enquêtes ou investigations sur les faits laissant supposer des violations des droits de l'enfant dans un État membre. En effet, l'article 45 de la Charte prévoit que le Comité pourra recourir à toute méthode appropriée pour enquêter sur toute question relevant de la CADBE. Il pourra, pour ce faire, demander aux États parties toute information pertinente sur l'application de la CADBE et recourir à toute méthode appropriée pour enquêter sur les mesures adoptées par les États, afin de mettre en œuvre les dispositions de la CADBE.

L'article 44 de la CADBE reconnaît la compétence du Comité d'experts en matière de communications 
individuelles. Ces communications, visant les États parties, pourront porter sur toute question traitée par la CADBE, et elles pourront être présentées par tout individu, groupe ou organisation non gouvernementale reconnue par l'UA, par un État membre, ou par l'ONU. La procédure de communications prévue par la CADBE constitue une avancée considérable par rapport à la Convention des Nations Unies relative aux droits de l'enfant. En effet, cette dernière ne prévoit aucune procédure contentieuse ou quasi contentieuse de contrôle et de sanction des violations des droits de l'enfant reconnus dans la Convention. Les seuls mécanismes prévus sont ceux axés sur la promotion des droits proclamés.

Toutefois, il faut souligner que la CADBE, à la différence de la Charte africaine des droits de l'homme et des peuples, ne contient aucune clause précisant les conditions de recevabilité et d'examen des communications individuelles qui pourront être présentées contre les États parties. Au titre de cette compétence, le Comité a reçu une plainte d'une ONG d'Ouganda et des préoccupations ont été exprimées par les membres du Comité au sujet de ce qui doit être fait avec cette communication. Il a été souligné, au cours de sa $6^{\mathrm{e}}$ session, qu'il s'agissait d'une question juridique et que le Comité n'avait encore reçu aucune directive sur la façon de traiter de telles questions. À l'issue d'un long débat, il a été décidé que le Secrétariat accuse réception de la communication en attendant que le Bureau du Conseiller juridique de l'UA dise si le Comité était mandaté pour recevoir et traiter une telle communication. Si le Bureau du Conseiller juridique l'affirme, le Comité examinera, d'une "façon informelle», la question au cours de sa $7^{\mathrm{e}}$ session.

Une telle interprétation est inquiétante quant à l'expertise juridique des membres du Comité et à la perception réelle de leur mission au regard de l'ensemble du système institutionnel de l'UA. Il est clair que le Comité a des attributions précises en matière d'examen des communications. Certes, les conditions de recevabilité n'ont pas été précisées par la CADBE. Toutefois, les membres du Comité devraient plutôt s'inspirer de la Charte africaine et du protocole créant la Cour pour aboutir à une harmonisation des conditions de recevabilité au sein du système africain de protection des droits de l'homme, au lieu de spéculer sur les éclairages du Bureau du Conseil juridique de l'UA. Il est clair que la « jurisprudence » de la Commission africaine des droits de l'homme pourrait être mise en exergue pour fixer les conditions de recevabilité et consolider ainsi ce «patrimoine commun» des procédures africaines en matière des droits de l'homme.

À l'instar des autres instances internationales et au regard des précédents déjà très nombreux dans le système africain, le Comité d'experts ne peut se saisir valablement d'une communication individuelle que lorsque les voies de recours internes auront été épuisées et qu'aucune autre «juridiction» internationale n'aura été saisie. Il s'agit donc d'une saisine exclusive qui interdit au requérant de tenter sa chance devant toutes les instances internationales et régionales au même moment, pour éviter ainsi de les mettre toutes en concurrence sur la même affaire. Ce passage obligatoire devant le juge interne est cependant perçu par de nombreux justiciables comme un préalable dissuasif dans la mesure où l'indépendance de la justice en Afrique n'est pas garantie.

Nous pensons que deux idées principales peuvent être développées sur ce point particulier.

Premièrement, la Commission africaine des droits de l'homme et des peuples s'est prononcée à de nombreuses occasions pour préciser qu'il «serait incorrect d'obliger les plaignants à user des voies de recours qui ne fonctionnent pas de façon impartiale et qui ne sont pas tenues de statuer conformément aux principes de droit. Le recours n'est ni adéquat ni efficace» (Communication 60 / 91, Affaire Constitutional Rights Project c. Nigeria), c'est-àdire que l'on ne saurait contraindre les plaignants à «épuiser des voies de recours internes qui, en termes pratiques, ne sont ni disponibles ni pratiques » (Communication 71 / 92, RADDHO c. Zambie). Enfin, l'on ne saurait épuiser les voies de recours internes sans une assistance judiciaire adéquate lorsqu'elle est nécessaire. Ainsi, «l'accusé devrait être représenté par un avocat de son choix. L'objectif de cette disposition est de garantir que l'accusé ait confiance en son avocat» (Communication 218/98, \$28-29). La situation est aggravante lorsque l'avocat de l'accusé est lui-même harcelé par le pouvoir. Ainsi dans l'affaire Ken Saro-Wiwa, la commission souligne que le fait de harceler les avocats, de les menacer dans leur vie privée ou professionnelle, jusqu'à les pousser à se retirer de l'affaire est une violation de la Charte africaine qui prive les plaignants d'une bonne administration de la justice ( $\$ 26-30)$. Il s'agit d'une confirmation de l'interprétation adoptée dans l'affaire Constitutional Rights Project c. Nigeria (Communication 87 / 93) où la Commission a conclu à la violation de la Charte dès lors que " pendant le procès, l'avocat de la défense a été harcelé et intimidé à tel point qu'il a été obligé de se retirer de la procédure ».

Le professeur Maurice Kamto pense qu'il faut cependant relativiser le caractère infranchissable que représenterait l'obstacle de la justice interne pour de nombreux justiciables. Il soutient qu'on ne peut pas se contenter de proclamer les déficiences connues de la justice en Afrique. Par-delà les problèmes réels, « il est juste de relever l'apathie des justiciables et l'inefficacité de leurs conseils ${ }^{19}$. Dans de nombreux pays africains, la reconnaissance judiciaire de l'applicabilité des conventions internationales n'a pas entraîné de la part des justiciables un engouement à s'y fonder pour obtenir la protection de leurs droits. Bien souvent, la méconnaissance par les justiciables des procédures judiciaires particulièrement complexes et formalistes entraîne une réaction de suspicion à l'égard de la justice.

Deuxièmement, la recevabilité de la communication devant le Comité d'experts suppose déjà que la requête

19. M. Kamto, «Charte africaine, instruments internationaux de protection des droits de l'homme, constitutions nationales : articulations et perspectives », in L'Application nationale de la Charte africaine des droits de l'homme et des peuples, p. 43. 
ait été acceptée comme telle par au moins une majorité des membres du Comité, faute de quoi elle ne serait pas inscrite sur le registre officiel des communications qui est le début de la procédure. De même, la saisine du Comité d'experts ne devrait pas se transformer en une entreprise calomnieuse contre les États membres et les principes de l'UA. Bien plus, la réunion cumulative des sept conditions de recevabilité définies à l'article 56 de la Charte africaine des droits de l'homme et des peuples serait obligatoire pour franchir le seuil de la recevabilité.

Quant au droit applicable, l'article 46 de la CADBE précise que « le Comité s'inspire du droit international relatif aux droits de l'homme [...] ainsi que des valeurs du patrimoine traditionnel et culturel africain ».

Lorsque le Comité d'experts pourrait constater que les violations ont eu lieu, il ferait des recommandations à l'État visé, afin qu'il s'assure qu'une enquête est menée sur ces allégations, que les victimes sont dédommagées (le cas échéant) et que des mesures sont prises pour éviter que cela ne se reproduise. Une telle investigation devrait éviter le renvoi de nombreuses requêtes souvent envoyées par des enfants vivant dans le désespoir et ne disposant pas d'un niveau suffisant pour comprendre toute la complexité des procédures devant une instance internationale.

\section{Les articulations avec les autres mécanismes de protection des droits de l'enfant}

Les relations naturelles entre la Commission ou la Cour africaine des droits de l'homme peuvent cependant masquer des conflits de compétence. D'où l'intérêt d'une rationalisation et d'une harmonisation des procédures et des termes de la gouvernance de ces organes pour avoir un système global renforcé et efficace. Pour le Comité d'experts, l'expérience du Comité des Nations Unies sera d'une grande utilité.

\section{a. L'articulation avec la Commission \\ et la Cour africaines des droits de l'homme}

Les mandats du Comité d'experts et de la Commission des droits de l'homme sont clairement définis dans les chartes les instituant. Malgré les similarités de leurs mandats, ceux du Comité se rapportent particulièrement aux droits de l'enfant, alors que ceux de la Commission sont en rapport avec les droits de l'homme et des peuples en général. Ainsi donc, il s'avère nécessaire pour le Comité et la Commission d'établir des relations complémentaires dans la réalisation de leurs mandats. Les modalités de cette étroite coopération devraient être examinées, et faire l'objet d'un consensus entre le Comité et la Commission.

En ce qui concerne la relation entre le Comité et la Cour africaine des droits de l'homme et des peuples, le Comité a reçu le mandat d'interpréter les dispositions de la CADBE, en cas de conflit au sujet de cette interprétation ou de l'application, la Cour a la compétence d'examiner les cas qui lui sont soumis, et de se prononcer selon le cas. De plus, il serait nécessaire que le Comité ait accès à la Cour. On peut également proposer que les secrétariats du Comité et de la Commission puissent travailler en étroite collaboration en vue d'harmoniser leurs activités.
Enfin, sur le plan procédural il est souhaitable que la lex specialis de la CADBE soit mise en valeur. La Charte africaine des droits de l'homme et des peuples a une portée plus générale, alors que la CADBE a une portée spécialisée. De ce fait, toutes les questions touchant les droits de l'enfant pourraient faire l'objet d'un renvoi préjudiciel devant le Comité d'experts par la Commission ou la Cour. De même, toutes les questions plus larges dépassant le cadre strict des droits de l'enfant pourraient être renvoyées par le Comité d'experts devant la Commission ou la Cour africaines des droits de l'homme et des peuples. Cette synergie et cette mutualisation des procédures permettraient un renforcement du système africain de protection des droits de l'homme. Dans ce sens, un secrétariat commun permettrait d'opérer une meilleure orientation des communications individuelles, à l'instar du système onusien au niveau du Haut-Commissariat à Genève.

\section{b. L'articulation avec le Comité des droits de l'enfant des Nations Unies}

Le Règlement intérieur du Comité d'experts précise que dans le cas où le rapport initial d'un État a déjà été examiné par le Comité des droits de l'enfant de l'ONU, "les observations finales et les recommandations du Comité seront prises en compte par le Comité africain au moment de la préparation de la liste de questions à soumettre au gouvernement concerné et lors de l'adoption de ses propres observations finales et recommandations ». L'un des membres du Comité d'experts, $\mathrm{M}^{\mathrm{me}}$ Nakpa Polo, a assisté à la $38^{\mathrm{e}}$ session du Comité des Nations Unies sur les droits de l'enfant à Genève, en qualité d'observateur. Le Comité des Nations Unies est disposé à fournir au Comité africain des experts toutes les informations et l'assistance requises. Il doit y avoir une synergie entre le Comité des experts et le Comité des Nations Unies, étant donné que le rapport qui sera soumis au Comité africain viendra compléter le rapport présenté au Comité des Nations Unies.

En outre, les directives générales concernant la forme et le contenu des rapports initiaux à présenter dans le cadre de l'article 44 (1) (a) de la CIDE ont largement inspiré les directives pour l'établissement des rapports des États parties à la CADBE. En effet, l'article 25 des directives autorise les États à «s'inspirer de ces rapports pour rédiger ceux à soumettre au Comité africain, en incorporant les éléments nouveaux et spécifiques à la Charte africaine». Il est à espérer que cette disposition, qui tend à limiter les lenteurs administratives inhérentes à la multiplication des obligations conventionnelles en matière de rapports, sera bien accueillie par les États et aura pour effet de les encourager à respecter les obligations découlant pour eux de la CADBE.

\section{B. Un dispositif aux capacités et perspectives limitées}

Le dispositif en cours de consolidation mérite d'être renforcé pour éviter un enlisement prévisible au regard 
des entraves déjà constatées et des défis titanesques qui l'attendent.

\section{L'action entravée du Comité des experts}

L'absence d'un Secrétariat permanent paraît à l'origine de certains dysfonctionnements du Comité d'experts, par exemple en ce qui concerne la publicité de ses sessions et sa coordination administrative. De même, le défaut de Secrétariat permanent, et donc de contact avec le personnel de l'Union africaine à Addis-Abeba, a été évoqué par certains membres du Comité comme ayant eu des conséquences, au cours de leurs premières années de mandat, sur les activités qu'ils auraient pu entreprendre au niveau international. Les États membres de l'UA donnent l'impression de ne pas se préoccuper du sort du Comité d'experts. Certes, il y a une inflation institutionnelle au sein de l'UA avec la prolifération des comités et organes techniques. Vu l'importance de la CADBE, la moindre des choses aurait été de doter le Comité d'experts d'un secrétariat véritablement opérationnel, au lieu de le confiner à l'étroit au sein de la Commission des affaires sociales.

Contrairement à ce que prescrit l'article 38 (5) de la CADBE, le Règlement intérieur définit les langues de travail du Comité comme étant l'anglais et le français. Aucun membre du Comité n'est, pour l'instant, ressortissant d'un pays de l'Afrique du Nord. Toutefois, la question peut être posée de savoir si la mise à l'écart de l'arabe et des autres langues officiels de l'UA ne va pas conduire l'Algérie, la Tunisie, la Libye et l'Égypte, y compris leurs populations et notamment les enfants d'Afrique du Nord, à se sentir moins concernés par les activités du Comité d'experts. Si des considérations matérielles légitimes peuvent être à la base de ce choix, la diversité culturelle, linguistique, mais aussi juridique de l'Afrique, impose malgré tout que les groupes majoritaires du continent soient représentés au sein du Comité. Le Comité étant un organe de l'UA, il se doit de respecter toutes les langues officielles de l'organisation.

\section{Le caractère titanesque des défis africains en matière de protection de l'enfant}

Même les bonnes nouvelles perdent de leur force quand on considère les difficultés que connaissent les enfants en Afrique. Malgré les modestes progrès dont ont bénéficié les enfants de certains pays d'Afrique au cours des années 1990, les enfants d'Afrique subsaharienne ont davantage de risques d'être malades que les enfants de toute autre région du monde. Ils ont par ailleurs moins de chances d'être scolarisés et risquent davantage de mourir avant d'atteindre l'âge de 5 ans. Les enfants africains et leurs parents, pris dans un engrenage de guerre, de maladies et de pauvreté toujours plus grand, se trouvent dans une mauvaise posture pour faire face aux défis de la mondialisation.

Les trois principales catastrophes qui entravent la jouissance des droits de l'enfant en Afrique sont le sida, la guerre et la pauvreté. Sur les 2,7 millions de séropositifs âgés de moins de 15 ans dans le monde en 2001, 2,4 millions se trouvaient en Afrique. Le nombre de personnes infectées par la tuberculose, étroitement liée au VIH / Sida, a également fait un bond et le nombre de cas en Afrique est passé de 16 pour 100000 en 1993 à 52 pour 100000 à la fin de 1999. Sur plus de 13 millions d'enfants dans le monde qui ont perdu un de leurs parents ou les deux à cause du sida, plus de 12 millions sont des Africains.

Les conflits ont également anéanti le progrès des droits de l'enfant en Afrique. Aucun conflit au monde n'a eu des retombées aussi catastrophiques sur les enfants que le conflit qui sévit dans l'est de la République démocratique du Congo, où l'on estime à 2,5 millions le nombre de décès dus aux hostilités qui ont commencé en 1998 (les victimes étant des femmes et des enfants en grande majorité). Les enfants ne sont pas uniquement ciblés par la violence, il arrive qu'ils commettent eux-mêmes des violences lorsqu'ils sont enrôlés de force aussi bien par l'armée du gouvernement que par les forces rebelles. Ils sont parfois contraints de commettre des atrocités contre des membres de leur propre famille

Enfin, le dernier obstacle à l'amélioration de la situation des droits de l'enfant en Afrique est la pauvreté. La Banque mondiale estime à environ 70 millions le nombre d'Africains qui, au cours des années 1990, sont allés grossir les rangs des indigents. Le nombre d'enfants et d'adultes africains qui arrivent tout juste à survivre est ainsi passé à 300 millions environ. Une étude menée en 1998 dans les pays où vivent $60 \%$ de la population africaine a conclu que plus de la moitié des personnes sur lesquelles l'enquête avait porté subsistaient avec des revenus d'à peine 67 cents par jour. Il n'est donc pas étonnant que les enfants africains n'aient pas réussi à progresser au même rythme que les enfants d'autres régions du monde.

Les membres du Comité d'experts doivent avoir à l'esprit cette trame des réalités africaines pour mieux se saisir des objectifs de la CADBE et mieux comprendre les limites d'une ambition qui en se voulant progressiste ne garantit pas pour autant un progrès certain.

Outre les conflits fratricides, le sida, le paludisme et la pauvreté, la vulnérabilité de l'enfant en Afrique est due, entre autres, à l'analphabétisme, aux résistances et pesanteurs socioculturelles de toutes sortes, à la démographie galopante et incontrôlée, à la corruption et l'absence de démocratie, au mépris des lois et conventions internationales considérées comme inutiles dans un contexte de précarité quotidienne et à l'insuffisante qualification des personnes impliquées dans les questions relatives aux enfants.

Les juridictions internes et la Cour africaine des droits de l'homme devraient participer à l'œuvre de socialisation de la CADBE et des autres instruments internationaux relatifs aux droits de l'enfant ${ }^{20}$. Au-delà des problèmes de formation et d'information des acteurs de la justice, c'est 
une invitation à mettre la $\mathrm{CADBE}$ au cour des pratiques judiciaires, car les États africains ne peuvent se résoudre à ignorer leurs propres engagements internationaux et proclamations officielles ${ }^{21}$. Certes, le discours politique ambiant semble accorder la priorité à la satisfaction des besoins essentiels des enfants (en particulier la santé et l'éducation) qui sont dans des conditions de pauvreté. Mais il est quasiment impossible de sortir de la pauvreté pour un enfant sans une réelle jouissance de l'ensemble de ses droits fondamentaux.

Le système africain de protection des droits de l'enfant offre non seulement des réelles possibilités de blâmer les États dont les comportements paraîtraient manifestement peu soucieux de la dignité humaine de l'enfant, mais il peut contribuer aussi à favoriser une réelle intégration des États africains par le droit sur la base des valeurs universelles partagées par la communauté internationale. L'attractivité du système africain de protection des droits de l'enfant reste à construire. Les voies d'accès à ce système peuvent apparaître escarpées pour de nombreuses victimes qui tenteront de les emprunter. Reste que le système a le mérite d'exister. Il contribue progressivement à l'émergence et la consolidation d'une protection régionale des droits de l'enfant en Afrique. Les limites et imperfections de la CADBE peuvent être surmontées avec une réelle volonté des États membres de l'Union africaine. Les arguments jadis évoqués sur les cultures africaines devraient être abandonnés, car l'universalité des droits de l'enfant n'est pas un obstacle à la diversité des cultures. Mais il existe des valeurs universelles sur lesquelles aucune régression ne peut être tolérée. Une telle évolution est souhaitable pour peu que l'Union africaine place les droits de l'enfant au cœur du panafricanisme qu'elle prétend réaliser pour préserver les enfants africains des fléaux de la guerre, de la peur et de la misère. 